Supplement of Clim. Past, 16, 1969-1986, 2020

https://doi.org/10.5194/cp-16-1969-2020-supplement

(c) Author(s) 2020. This work is distributed under

the Creative Commons Attribution 4.0 License.

(c) (1)

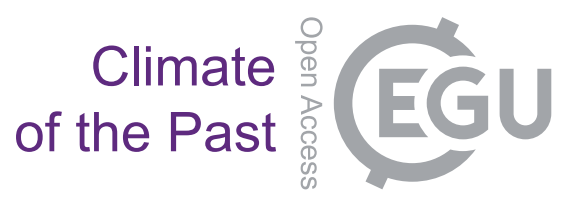

Supplement of

\title{
Dynamics of primary productivity in the northeastern Bay of Bengal over the last 26000 years
}

\section{Xinquan Zhou et al.}

Correspondence to: Xinquan Zhou (xinquan.zhou@universite-paris-saclay.fr)

The copyright of individual parts of the supplement might differ from the CC BY 4.0 License. 


\section{Supplementary Figures}

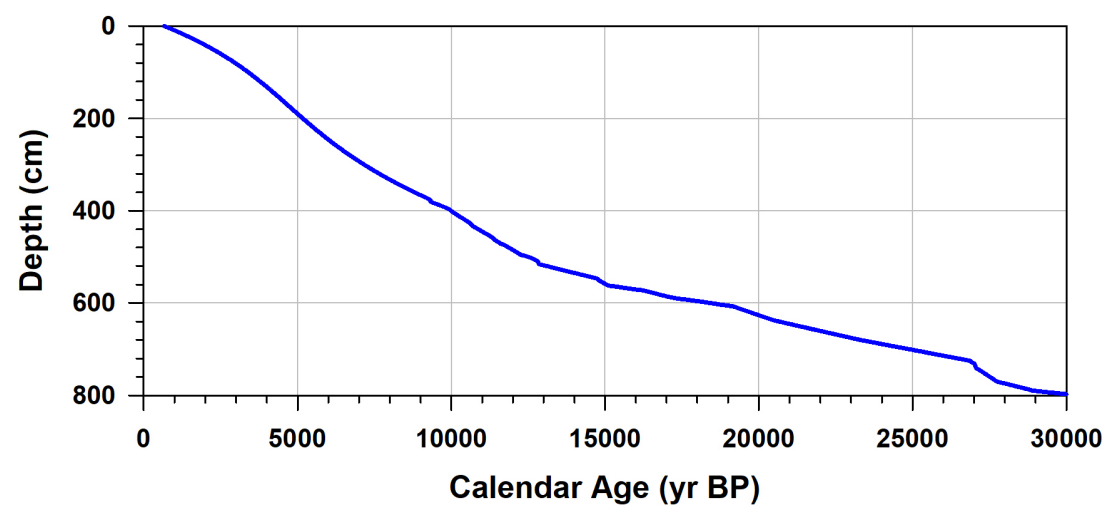

Fig. S1. Age model of core MD77-176. This age model was a tuned model constructed by Marzin et al., (2013).

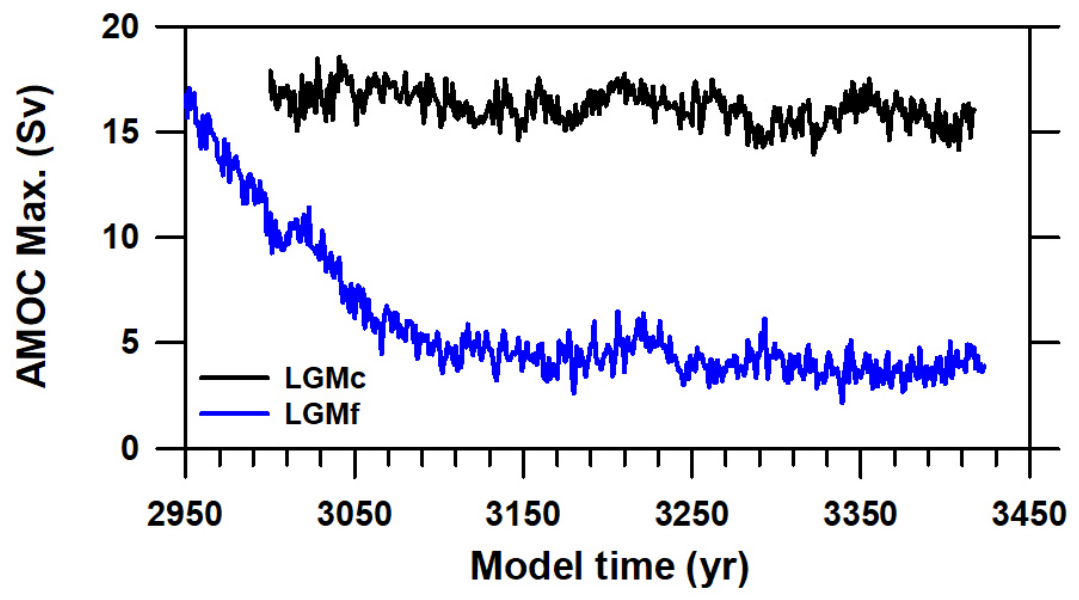

Fig. S2. Changes of the maximum in the AMOC stream function below $500 \mathrm{~m}$ (AMOC strength) in LGMc and LGMf experiments run with IPSL-CM5A-LR for nearly 500 model years, which are labeled from 2950 to 3450 in the numerical experiments. 


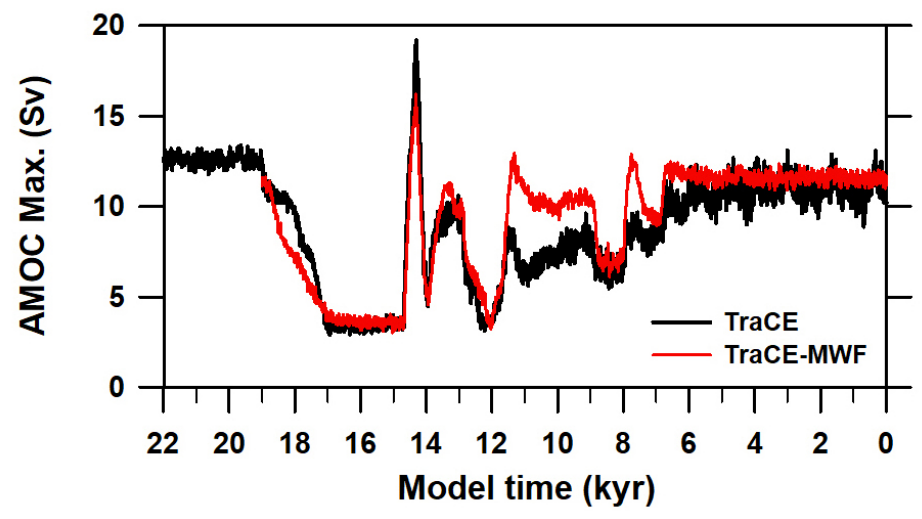

Fig. S3. Changes of the maximum in the AMOC stream function below $500 \mathrm{~m}$ (AMOC strength) in the full TraCE21 simulation over the $22 \mathrm{kyr}$ model time and melt water of ice sheets single forcing simulation (MWF) over the 19 kyr model time. Significant variations are found between 19 and $11 \mathrm{kyr}$ (the deglaciation)
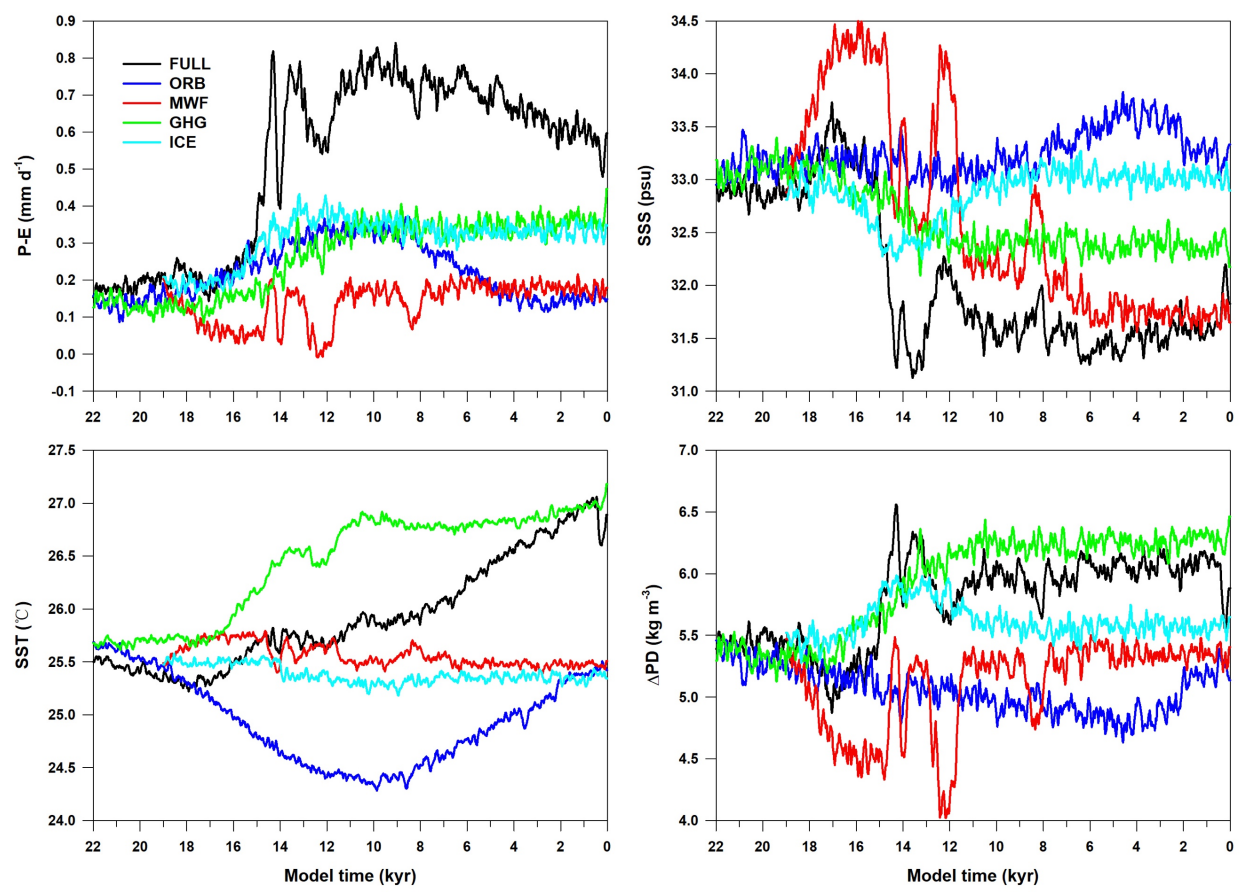

Fig. S4. Annual mean results of net precipitation (P-E), SSS, SST and potential density difference between 200 and $5 \mathrm{~m}(\triangle \mathrm{PD})$ in full TraCE-21 simulation, and single forcing simulations (section 3.3.2): orbital insolation (ORB), green-house gas concentration (GHG), meltwater flux (MWF) and ice sheet (ICE). Over the last deglaciation time from 19 to $11 \mathrm{kyr}$, the results show that the millennial-scale variations of net precipitation, SSS and stratification are mainly contributed by MWF forcing which moderated AMOC strength. The changes in other single forcing experiment show no obvious millennial-scale variations during the last deglaciation. The changes of SST during the deglaciation is very limited. 


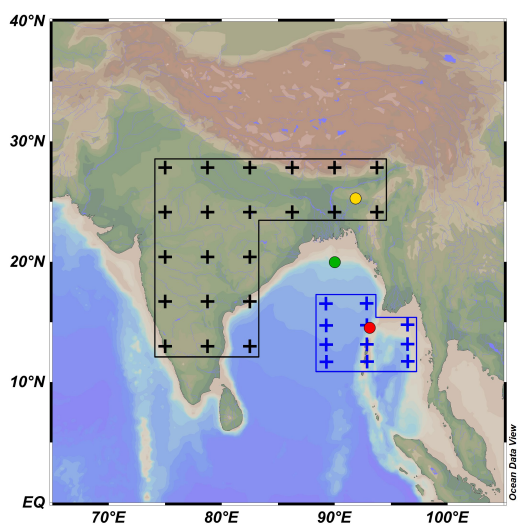

Fig. S5. Geographic map of South Asia, the Bay of Bengal and the Andaman Sea, the The map was created by the Ocean Data View software (CReiner Schlitzer, Alfred Wegener Institute) with its built-in global high resolution bathymetric data (GlobHR). The grids of data extracting. Black cross are grids for TraCE-21 atmospheric outputs. Blue grids are for TraCE-21 oceanic outputs. The yellow, green and red dots mark the locations of Mawmluh Cave, core SO188-342 and core MD77-176 respectively. 


\section{Supplementary data}

Relative abundances (\%) of main coccolith species of core MD77-176. Emi = Emiliania huxleyi, Gep $=$ Gephyrocapsa spp., Fp = Florisphaera profunda .

\begin{tabular}{|c|c|c|c|c|c|}
\hline Depth (cm) & Age (ka) & Emi \% & Gep \% & Fp \% & Other \% \\
\hline 10.5 & 1.04 & 4.72 & 15.41 & 75.63 & 4.25 \\
\hline 22.5 & 1.44 & 4.71 & 18.31 & 73.13 & 3.85 \\
\hline 25.5 & 1.53 & 4.12 & 15.36 & 78.74 & 1.78 \\
\hline 28.5 & 1.63 & 5.14 & 20.55 & 70.88 & 3.43 \\
\hline 31.5 & 1.72 & 4.16 & 16.34 & 75.07 & 4.43 \\
\hline 34.5 & 1.81 & 6.18 & 15.29 & 74.95 & 3.58 \\
\hline 37.5 & 1.89 & 4.2 & 22.49 & 69.81 & 3.5 \\
\hline 40.5 & 1.98 & 4.52 & 17.31 & 75.16 & 3.01 \\
\hline 43.5 & 2.06 & 4.24 & 9.32 & 83.90 & 2.54 \\
\hline 46.5 & 2.15 & 5.67 & 16.21 & 74.23 & 3.89 \\
\hline 49.5 & 2.22 & 4.43 & 17.86 & 73.29 & 4.43 \\
\hline 52.5 & 2.3 & 5.11 & 10.1 & 81.67 & 3.12 \\
\hline 55.5 & 2.38 & 3.21 & 9.27 & 86.63 & 0.89 \\
\hline 58.5 & 2.46 & 6.62 & 8.62 & 83.23 & 1.54 \\
\hline 61.5 & 2.54 & 5.13 & 18.04 & 73.61 & 3.23 \\
\hline 64.5 & 2.61 & 6.38 & 20.5 & 68.25 & 4.88 \\
\hline 67.5 & 2.68 & 5.02 & 15.94 & 74.92 & 4.12 \\
\hline 70.5 & 2.75 & 6.7 & 11.9 & 78.52 & 2.87 \\
\hline 73.5 & 2.82 & 4.16 & 11.76 & 81.21 & 2.87 \\
\hline 76.5 & 2.89 & 5.28 & 9.28 & 82.09 & 3.35 \\
\hline 79.5 & 2.96 & 6.44 & 13.37 & 76.98 & 3.22 \\
\hline 82.5 & 3.03 & 5.39 & 11.21 & 80.71 & 2.7 \\
\hline 85.5 & 3.09 & 5.21 & 10.42 & 81.51 & 2.85 \\
\hline 88.5 & 3.16 & 4.69 & 16.28 & 73.10 & 5.93 \\
\hline 91.5 & 3.22 & 6.93 & 15.47 & 73.76 & 3.84 \\
\hline 94.5 & 3.28 & 6.78 & 12.25 & 76.93 & 4.04 \\
\hline 97.5 & 3.35 & 6.22 & 15.05 & 73.76 & 4.98 \\
\hline 100.5 & 3.41 & 5.12 & 12.4 & 79.54 & 2.94 \\
\hline 103.5 & 3.47 & 5.93 & 10.51 & 79.48 & 4.08 \\
\hline
\end{tabular}




\begin{tabular}{|c|c|c|c|c|c|}
\hline Depth (cm) & Age (ka) & Emi \% & Gep \% & Fp \% & Other \% \\
\hline 106.5 & 3.53 & 5.65 & 14.03 & 77.91 & 2.41 \\
\hline 109.5 & 3.58 & 2.79 & 14.9 & 78.83 & 3.48 \\
\hline 112.5 & 3.64 & 4.19 & 16.4 & 75.70 & 3.72 \\
\hline 115.5 & 3.7 & 3.82 & 11.01 & 82.36 & 2.81 \\
\hline 118.5 & 3.76 & 5.35 & 9.67 & 80.32 & 4.66 \\
\hline 121.5 & 3.81 & 5.8 & 19.62 & 71.10 & 3.48 \\
\hline 124.5 & 3.87 & 5.07 & 14.68 & 77.04 & 3.21 \\
\hline 127.5 & 3.92 & 4.72 & 9.55 & 82.22 & 3.51 \\
\hline 130.5 & 3.98 & 5.35 & 8.96 & 83.16 & 2.54 \\
\hline 133.5 & 4.03 & 4.43 & 10.76 & 82.03 & 2.78 \\
\hline 136.5 & 4.08 & 5.26 & 11.89 & 80.69 & 2.17 \\
\hline 139.5 & 4.14 & 2.72 & 11.93 & 82.75 & 2.59 \\
\hline 142.5 & 4.19 & 4.81 & 15.78 & 74.97 & 4.44 \\
\hline 145.5 & 4.24 & 4.48 & 13.34 & 78.00 & 4.18 \\
\hline 148.5 & 4.29 & 5.18 & 15.15 & 74.37 & 5.3 \\
\hline 151.5 & 4.35 & 4.18 & 14.73 & 78.54 & 2.55 \\
\hline 154.5 & 4.4 & 5.68 & 13.46 & 77.18 & 3.68 \\
\hline 157.5 & 4.45 & 8.1 & 9.4 & 80.12 & 2.38 \\
\hline 160.5 & 4.5 & 6.48 & 15.31 & 75.03 & 3.18 \\
\hline 163.5 & 4.55 & 4.3 & 13.86 & 78.26 & 3.58 \\
\hline 166.5 & 4.6 & 4.27 & 11.38 & 80.09 & 4.27 \\
\hline 169.5 & 4.65 & 4.52 & 15.93 & 75.41 & 4.14 \\
\hline 172.5 & 4.7 & 4.31 & 8.49 & 84.33 & 2.87 \\
\hline 175.5 & 4.75 & 5.35 & 13.75 & 77.59 & 3.31 \\
\hline 178.5 & 4.8 & 6.02 & 11.08 & 77.96 & 4.95 \\
\hline 181.5 & 4.85 & 4.35 & 13.78 & 77.39 & 4.47 \\
\hline 184.5 & 4.91 & 4.62 & 14.5 & 77.52 & 3.36 \\
\hline 187.5 & 4.96 & 5.76 & 7.75 & 83.28 & 3.21 \\
\hline 190.5 & 5.01 & 6.47 & 10.27 & 79.06 & 4.2 \\
\hline 193.5 & 5.06 & 5.42 & 16.03 & 73.81 & 4.74 \\
\hline 196.5 & 5.11 & 5.61 & 9.27 & 81.71 & 3.41 \\
\hline 199.5 & 5.16 & 4.82 & 10.81 & 79.69 & 4.69 \\
\hline 202.5 & 5.21 & 4.46 & 11.01 & 81.91 & 2.62 \\
\hline 205.5 & 5.27 & 5.53 & 7.1 & 84.34 & 3.03 \\
\hline
\end{tabular}




\begin{tabular}{|c|c|c|c|c|c|}
\hline Depth (cm) & Age (ka) & Emi \% & Gep \% & Fp \% & Other \% \\
\hline 208.5 & 5.32 & 6.74 & 9.77 & 78.37 & 5.12 \\
\hline 211.5 & 5.37 & 5.7 & 12.44 & 77.79 & 4.07 \\
\hline 214.5 & 5.42 & 4.53 & 12.69 & 77.20 & 5.57 \\
\hline 217.5 & 5.48 & 5.95 & 15.33 & 73.98 & 4.74 \\
\hline 220.5 & 5.53 & 7.42 & 13.39 & 74.35 & 4.84 \\
\hline 223.5 & 5.58 & 6.23 & 11.25 & 78.35 & 4.18 \\
\hline 226.5 & 5.64 & 6.33 & 12.85 & 76.61 & 4.22 \\
\hline 229.5 & 5.69 & 5.82 & 12.55 & 76.22 & 5.41 \\
\hline 232.5 & 5.75 & 3.6 & 6.56 & 87.77 & 2.06 \\
\hline 235.5 & 5.8 & 5.74 & 8.57 & 81.99 & 3.7 \\
\hline 238.5 & 5.86 & 5.86 & 9.01 & 81.65 & 3.47 \\
\hline 241.5 & 5.92 & 5.7 & 8.94 & 82.41 & 2.95 \\
\hline 244.5 & 5.98 & 4.84 & 8.44 & 83.33 & 3.4 \\
\hline 247.5 & 6.03 & 4.14 & 11.31 & 80.05 & 4.5 \\
\hline 250.5 & 6.09 & 4.43 & 10.11 & 82.39 & 3.07 \\
\hline 253.5 & 6.15 & 5.42 & 11.16 & 78.98 & 4.44 \\
\hline 256.5 & 6.21 & 4.92 & 6.93 & 85.74 & 2.41 \\
\hline 259.5 & 6.27 & 3.32 & 8.31 & 85.80 & 2.57 \\
\hline 262.5 & 6.33 & 4.86 & 8.46 & 82.56 & 4.12 \\
\hline 265.5 & 6.4 & 4.36 & 12.85 & 78.54 & 4.25 \\
\hline 268.5 & 6.46 & 3.3 & 9.9 & 83.51 & 3.3 \\
\hline 271.5 & 6.52 & 6.97 & 13.18 & 74.28 & 5.57 \\
\hline 274.5 & 6.59 & 4.62 & 9.06 & 84.01 & 2.31 \\
\hline 277.5 & 6.65 & 3.71 & 15.03 & 77.64 & 3.62 \\
\hline 280.5 & 6.72 & 4.03 & 13.89 & 77.51 & 4.57 \\
\hline 283.5 & 6.78 & 4.22 & 12.76 & 78.33 & 4.68 \\
\hline 286.5 & 6.85 & 3.06 & 18.03 & 73.66 & 5.25 \\
\hline 289.5 & 6.92 & 4.45 & 8.99 & 82.59 & 3.97 \\
\hline 292.5 & 6.99 & 4.86 & 10.8 & 79.58 & 4.76 \\
\hline 295.5 & 7.06 & 2.49 & 8.28 & 85.33 & 3.91 \\
\hline 298.5 & 7.13 & 4.08 & 7.7 & 85.28 & 2.94 \\
\hline 301.5 & 7.2 & 5.26 & 12.77 & 77.22 & 4.76 \\
\hline 304.5 & 7.27 & 3.28 & 8.26 & 84.19 & 4.27 \\
\hline 307.5 & 7.35 & 4.98 & 8.78 & 83.36 & 2.88 \\
\hline
\end{tabular}




\begin{tabular}{|c|c|c|c|c|c|}
\hline Depth (cm) & Age (ka) & Emi \% & Gep \% & Fp \% & Other \% \\
\hline 310.5 & 7.42 & 2.84 & 9.88 & 82.14 & 5.14 \\
\hline 313.5 & 7.5 & 4.2 & 7.65 & 83.33 & 4.81 \\
\hline 316.5 & 7.57 & 3.91 & 8.77 & 82.46 & 4.86 \\
\hline 319.5 & 7.65 & 3.05 & 12.75 & 79.81 & 4.38 \\
\hline 322.5 & 7.73 & 3.95 & 8.67 & 84.08 & 3.29 \\
\hline 325.5 & 7.81 & 3.18 & 8.54 & 84.37 & 3.91 \\
\hline 328.5 & 7.89 & 3.65 & 9.55 & 84.76 & 2.04 \\
\hline 334.5 & 7.97 & 2.18 & 7.94 & 87.39 & 2.48 \\
\hline 334.5 & 8.05 & 4.9 & 9.93 & 80.51 & 4.66 \\
\hline 337.5 & 8.14 & 3.27 & 13.61 & 78.64 & 4.49 \\
\hline 340.5 & 8.22 & 4.35 & 12.11 & 80.00 & 3.54 \\
\hline 343.5 & 8.31 & 5.59 & 14.85 & 74.26 & 5.29 \\
\hline 346.5 & 8.4 & 4.81 & 13.91 & 76.46 & 4.81 \\
\hline 349.5 & 8.49 & 3.02 & 12.76 & 80.11 & 4.12 \\
\hline 352.5 & 8.58 & 2.2 & 10.3 & 83.38 & 4.12 \\
\hline 355.5 & 8.66 & 3.48 & 17.58 & 73.49 & 5.46 \\
\hline 358.5 & 8.76 & 3.16 & 13.54 & 77.72 & 5.57 \\
\hline 361.5 & 8.85 & 3.51 & 15.2 & 75.72 & 5.58 \\
\hline 364.5 & 8.94 & 4.33 & 11.94 & 81.19 & 2.54 \\
\hline 367.5 & 9.04 & 3.92 & 13.52 & 78.20 & 4.36 \\
\hline 370.5 & 9.14 & 3.2 & 13.11 & 79.12 & 4.57 \\
\hline 373.5 & 9.23 & 2.35 & 20.12 & 72.59 & 4.94 \\
\hline 376.5 & 9.29 & 3.96 & 17.91 & 73.42 & 4.7 \\
\hline 379.5 & 9.32 & 2.17 & 14.65 & 79.75 & 3.44 \\
\hline 382.5 & 9.41 & 4.62 & 18.35 & 71.15 & 5.88 \\
\hline 385.5 & 9.52 & 2.87 & 17.65 & 74.03 & 5.45 \\
\hline 388.5 & 9.63 & 1.62 & 12.48 & 82.33 & 3.57 \\
\hline 391.5 & 9.74 & 1.26 & 17.46 & 76.12 & 5.17 \\
\hline 394.5 & 9.86 & 1.64 & 15.22 & 80.20 & 2.95 \\
\hline 397.5 & 9.93 & 3.36 & 15.75 & 76.46 & 4.42 \\
\hline 400.5 & 10 & 2.63 & 15.46 & 76.97 & 4.95 \\
\hline 403.5 & 10.06 & 1.28 & 18.07 & 75.96 & 4.69 \\
\hline 406.5 & 10.13 & 1.45 & 16.96 & 78.03 & 3.55 \\
\hline 409.5 & 10.2 & 1.59 & 16.53 & 77.38 & 4.5 \\
\hline
\end{tabular}




\begin{tabular}{|c|c|c|c|c|c|}
\hline Depth (cm) & Age (ka) & Emi \% & Gep \% & Fp \% & Other \% \\
\hline 412.5 & 10.27 & 0.72 & 13.98 & 82.26 & 3.05 \\
\hline 415.5 & 10.34 & 1.66 & 15.79 & 76.32 & 6.23 \\
\hline 418.5 & 10.41 & 2.32 & 17.2 & 74.39 & 6.1 \\
\hline 421.5 & 10.49 & 1.91 & 17.85 & 74.25 & 5.99 \\
\hline 427.5 & 10.63 & 1.65 & 17.3 & 75.12 & 5.93 \\
\hline 433.5 & 10.71 & 0.19 & 17.59 & 78.97 & 3.25 \\
\hline 436.5 & 10.78 & 0.58 & 16.38 & 77.46 & 5.59 \\
\hline 439.5 & 10.86 & 0.54 & 22.26 & 73.97 & 3.23 \\
\hline 442.5 & 10.94 & 0.82 & 19.31 & 73.98 & 5.89 \\
\hline 445.5 & 11.01 & 0.88 & 20.14 & 75.48 & 3.5 \\
\hline 448.5 & 11.09 & 1.05 & 19.76 & 74.13 & 5.07 \\
\hline 451.5 & 11.17 & 2.17 & 18.79 & 75.16 & 3.88 \\
\hline 457.5 & 11.33 & 2.14 & 18.35 & 75.23 & 4.28 \\
\hline 466.5 & 11.5 & 2.25 & 19.69 & 74.27 & 3.8 \\
\hline 475.5 & 11.76 & 3.01 & 18.19 & 75.79 & 3.01 \\
\hline 478.5 & 11.89 & 3.85 & 16.92 & 76.72 & 2.51 \\
\hline 481.5 & 12.01 & 4.56 & 21.1 & 70.72 & 3.61 \\
\hline 496.5 & 12.3 & 3.77 & 14.5 & 78.15 & 3.58 \\
\hline 499.5 & 12.43 & 2.09 & 14.92 & 77.88 & 5.1 \\
\hline 505.5 & 12.69 & 4.36 & 12.89 & 79.67 & 3.09 \\
\hline 517.5 & 12.95 & 3.75 & 13.04 & 78.85 & 4.35 \\
\hline 523.5 & 13.3 & 2.05 & 13.89 & 77.05 & 7.02 \\
\hline 526.5 & 13.48 & 2.22 & 14.99 & 78.36 & 4.43 \\
\hline 529.5 & 13.66 & 2.79 & 10.12 & 81.15 & 5.93 \\
\hline 532.5 & 13.85 & 1.9 & 10.58 & 82.62 & 4.9 \\
\hline 535.5 & 14.03 & 0.95 & 5.52 & 92.90 & 0.63 \\
\hline 538.5 & 14.21 & 2.97 & 8.76 & 85.45 & 2.82 \\
\hline 541.5 & 14.4 & 7.67 & 12.7 & 75.13 & 4.5 \\
\hline 544.5 & 14.58 & 11.42 & 8.98 & 72.30 & 7.31 \\
\hline 547.5 & 14.71 & 6.79 & 8.81 & 82.02 & 2.39 \\
\hline 550.5 & 14.79 & 12.92 & 8.99 & 72.89 & 5.2 \\
\hline 553.5 & 14.87 & 11.59 & 11.36 & 64.12 & 12.94 \\
\hline 556.5 & 14.95 & 21.04 & 8.49 & 62.93 & 7.53 \\
\hline 559.5 & 15.04 & 19.03 & 12.01 & 61.20 & 7.76 \\
\hline
\end{tabular}




\begin{tabular}{|c|c|c|c|c|c|}
\hline Depth (cm) & Age (ka) & Emi \% & Gep \% & Fp \% & Other \% \\
\hline 562.5 & 15.15 & 20 & 9.79 & 62.70 & 7.52 \\
\hline 568.5 & 15.75 & 12.65 & 13.42 & 70.43 & 3.5 \\
\hline 571.5 & 16.04 & 12.96 & 10.56 & 70.56 & 5.92 \\
\hline 574.5 & 16.34 & 13.4 & 11.96 & 69.38 & 5.26 \\
\hline 583.5 & 16.74 & 16.99 & 7.58 & 69.32 & 6.11 \\
\hline 586.5 & 17.04 & 10.24 & 8.03 & 77.51 & 4.22 \\
\hline 589.5 & 17.34 & 15.93 & 7.66 & 72.98 & 3.43 \\
\hline 592.5 & 17.64 & 11.14 & 13.2 & 72.49 & 3.16 \\
\hline 595.5 & 17.94 & 11.05 & 9.65 & 74.41 & 4.9 \\
\hline 598.5 & 18.25 & 8.69 & 11.69 & 74.25 & 5.37 \\
\hline 601.5 & 18.55 & 12.55 & 10.65 & 73.13 & 3.68 \\
\hline 604.5 & 18.86 & 1.73 & 17.85 & 77.12 & 3.29 \\
\hline 607.5 & 19.17 & 3.31 & 13.07 & 77.70 & 5.92 \\
\hline 610.5 & 19.3 & 9.35 & 7.57 & 77.87 & 5.21 \\
\hline 613.5 & 19.43 & 6.89 & 10.48 & 78.14 & 4.49 \\
\hline 616.5 & 19.56 & 9.72 & 6.17 & 80.86 & 3.24 \\
\hline 619.5 & 19.69 & 7.19 & 8.82 & 80.72 & 3.27 \\
\hline 622.5 & 19.82 & 4.55 & 8.39 & 85.84 & 1.22 \\
\hline 625.5 & 19.95 & 7.46 & 8.96 & 79.55 & 4.03 \\
\hline 628.5 & 20.09 & 11.78 & 10.65 & 72.56 & 5.01 \\
\hline 631.5 & 20.22 & 6.45 & 10.89 & 78.63 & 4.03 \\
\hline 634.5 & 20.35 & 8.08 & 10.86 & 76.82 & 4.24 \\
\hline 637.5 & 20.49 & 12.66 & 11.96 & 67.45 & 7.93 \\
\hline 640.5 & 20.68 & 11.32 & 11.17 & 72.28 & 5.23 \\
\hline 643.5 & 20.88 & 8.2 & 10.43 & 77.05 & 4.32 \\
\hline 646.5 & 21.07 & 14.79 & 13.42 & 65.37 & 6.42 \\
\hline 649.5 & 21.27 & 5.14 & 10.45 & 82.26 & 2.16 \\
\hline 652.5 & 21.47 & 6.8 & 6.8 & 83.27 & 3.12 \\
\hline 655.5 & 21.67 & 9.65 & 9.83 & 77.37 & 3.15 \\
\hline 658.5 & 21.86 & 14.51 & 7.61 & 73.56 & 4.31 \\
\hline 661.5 & 22.06 & 9.02 & 9.8 & 79.02 & 2.16 \\
\hline 664.5 & 22.26 & 11.15 & 12.18 & 72.73 & 3.95 \\
\hline 667.5 & 22.46 & 9.63 & 17.43 & 69.72 & 3.21 \\
\hline 670.5 & 22.66 & 10.1 & 10.8 & 75.74 & 3.37 \\
\hline
\end{tabular}




\begin{tabular}{cccccc}
\hline Depth (cm) & Age (ka) & Emi \% & Gep \% & Fp \% & Other \% \\
\hline 673.5 & 22.87 & 17.7 & 13.1 & 65.31 & 3.89 \\
676.5 & 23.07 & 17.1 & 14.35 & 64.58 & 3.97 \\
679.5 & 23.27 & 13.32 & 16.07 & 68.08 & 2.54 \\
682.5 & 23.5 & 8.06 & 8.78 & 81.54 & 1.61 \\
685.5 & 23.73 & 14.67 & 10.09 & 72.71 & 2.52 \\
688.5 & 23.97 & 9.76 & 11.45 & 74.75 & 4.04 \\
691.5 & 24.2 & 6.33 & 9.31 & 83.05 & 1.3 \\
694.5 & 24.44 & 7.17 & 10.48 & 78.68 & 3.68 \\
697.5 & 24.68 & 10.66 & 11.44 & 73.51 & 4.39 \\
700.5 & 24.91 & 4.95 & 10.54 & 81.31 & 3.19 \\
703.5 & 25.15 & 13.81 & 12.29 & 69.65 & 4.25 \\
706.5 & 25.39 & 12.06 & 11.45 & 71.76 & 4.73 \\
709.5 & 25.63 & 16.4 & 14.27 & 61.20 & 8.13 \\
\hline
\end{tabular}

